\title{
Influência da cultura anterior e da compactação do solo na absorção de macronutrientes em soja ${ }^{(1)}$
}

\author{
Rosemeire Helena da Silva(2) e Ciro Antonio Rosolem ${ }^{(3)}$
}

\begin{abstract}
Resumo - O objetivo deste trabalho foi avaliar a concentração e a absorção de macronutrientes em soja cultivada em subseqüência a sete espécies vegetais em solo com diferentes níveis de compactação. O experimento foi conduzido em vasos contendo amostras de um Latossolo Vermelho, textura francoarenosa, com camada de 3,5 cm compactada até as densidades de 1,12, 1,36 e 1,60 $\mathrm{Mg} \mathrm{m}^{-3}$, em que se cultivou anteriormente aveia-preta, guandu, milheto, mucuna-preta, soja, sorgo granífero, tremoço azul, mantendo-se um tratamento sem planta (pousio). Essas espécies se desenvolveram por 37 a 39 dias, foram cortadas ao nível do solo, picadas em partículas de, aproximadamente, $3 \mathrm{~cm}$, e deixadas sobre a superfície do vaso por 40 dias. As plantas foram colhidas 28 dias após a emergência. Avaliou-se a produção de matéria seca, concentração e acúmulo de macronutrientes na parte aérea das plantas. A compactação do solo em subsuperfície diminui a nutrição da soja. A nutrição da soja é beneficiada quando cultivada em sucessão a plantas de cobertura no solo. O pousio antecedendo a cultura da soja não é recomendado como meio de reduzir os efeitos da compactação do solo.
\end{abstract}

Termos para indexação: Glycine max, nutrição das plantas, densidade do solo, adubação verde.

\section{Effect of the previous crop and of soil compaction on macronutrient uptake by soybean}

\begin{abstract}
The effect of seven species cropped just before soybean, as well as the effect of soil compaction on macronutrient uptake by soybean were evaluated. The experiment was conducted in pots containing a Red Latosol (Acrortox, loamy sand), and the pots had a layer $3.5 \mathrm{~cm}$ thick and $15 \mathrm{~cm}$ deep compacted to $1.12,1.36$ and $1.60 \mathrm{Mg} \mathrm{m}^{-3}$. Before soybean, the pots were cropped to black oat, pigeon pea, pearl millet, black mucuna, soybean, grain sorghum and lupin, plus a treatment without plants. These species were grown for 37 to 39 days, when they were cut at soil level, pricked into particles of approximately $3 \mathrm{~cm}$ length, and left on the soil surface for 40 days. After this, soybean was planted in the pots and grown for 28 days after plant emergence. The shoot dry matter weight, concentration and accumulation were evaluated. The subsoil compaction decreased the nutrition of soybean. The nutrition of soybean is benefited when in sequence to soil cover crops. The soil without plants is not recommended as well as method to reduce the effects of the soil compaction.
\end{abstract}

Index terms: Glycine max, plant nutrition, soil density, green manures.

\section{Introdução}

A rotação de culturas tem sido destacada por promover benefícios, como proteção do solo contra ero-

\footnotetext{
(1) Aceito para publicação em 17 de novembro de 2000 .

Extraído da Dissertação de Mestrado apresentada pelo primeiro autor à Universidade Estadual Paulista (Unesp), Botucatu, SP. Financiado pela Fapesp.

(2) Unesp, Faculdade de Ciências Agronômicas (FCA), Dep. de Produção Vegetal, Caixa Postal 237, CEP 18603-970 Botucatu, SP. Bolsista da Fapesp. E-mail: meire@fca.unesp.br

(3)Unesp, FCA, Dep. de Produção Vegetal. E-mail rosolem@fca.unesp.br
}

são, elevação da taxa de infiltração e aumento da capacidade de retenção de água, incremento da capacidade de reciclagem e mobilização de nutrientes lixiviados ou pouco solúveis em camadas mais profundas do solo, além de outros benefícios (Pinto \& Crestana, 1998). Entre estes, a reciclagem de nutrientes é uma característica importante, pois a quantidade de resíduos culturais sobre a superfície do solo pode afetar a produção das culturas seguintes (Jessop \& Stewart, 1983; Lynch, 1984); deve ser adotado um sistema de rotação de culturas adequado.

Outro aspecto a ser destacado é que, com a decomposição de resíduos vegetais, pode ocorrer 
maior concentração de nutrientes na camada superficial do solo. Dessa forma, em solos compactados, em virtude do impedimento físico ao crescimento de raízes, a maior disponibilidade de nutrientes na camada acima da compactada pode proporcionar um melhor desenvolvimento da cultura seguinte. Assim, espécies que possuam sistema radicular profundo e ramificado podem retirar nutrientes de camadas subsuperficiais, e liberá-los gradualmente nas camadas superficiais, durante o processo de decomposição (Fiorin, 1999).

Segundo Marschner (1986), dependendo do tipo de solo e da umidade, em densidades de até 1,4 $\mathrm{Mg} \mathrm{m}^{-3}$, o impedimento ao crescimento radicular não está correlacionado com a inibição da absorção de nutrientes, e pode ser atribuído ao aumento no poder-tampão do solo em relação aos nutrientes, ao aumento na densidade do solo (Silberbush et al., 1983), e aos reguladores de crescimento produzidos nas raízes. Pode ainda haver algum mensageiro químico envolvido (Bengough \& Young, 1993).

Este trabalho teve por objetivo avaliar a concentração e o acúmulo de macronutrientes na parte aérea da soja cultivada sobre resíduos de várias espécies vegetais e em pousio, em solo com camada compactada.

\section{Material e Métodos}

O experimento foi conduzido em casa de vegetação, na Faculdade de Ciências Agronômicas, Unesp, Campus de Botucatu, SP.

O solo utilizado foi proveniente da camada arável de um Latossolo Vermelho, textura franco arenosa, com 690, 150 e $160 \mathrm{~g} \mathrm{~kg}^{-1}$ de areia, silte e argila, respectivamente, e os resultados de sua análise química indicaram valores de $\mathrm{pH}$ em $\mathrm{CaCl}_{2}$ de 5,3; $3 \mu \mathrm{g} \mathrm{cm}^{-3}$ de P; 0,7, 13 e 9 mmol $_{\mathrm{c}} \mathrm{dm}^{-3} \mathrm{de}$ $\mathrm{K}, \mathrm{Ca}$ e Mg, respectivamente, e saturação por bases de $51 \%$.

O preparo do solo constou das seguintes etapas: peneiramento em malha de $4 \mathrm{~mm}$; calagem com $0,650 \mathrm{~g}$ por vaso de calcário com PRNT $100 \%$ (32\% de $\mathrm{CaO}$ e $18 \%$ de $\mathrm{MgO}$ ), para elevar a saturação por bases a $70 \%$; e adubação com P, K, Zn e B a 150, 120, 1,0 e 0,5 mg cm${ }^{-3}$, respectivamente.

O experimento foi instalado em esquema fatorial $3 \times 8$, inteiramente casualizado, com quatro repetições, no qual os tratamentos constaram de três densidades do solo: 1,12, 1,36 e $1,60 \mathrm{Mg} \mathrm{m}^{-3}$ e oito manejos: aveia-preta (Avena strigosa) cv. Comum, guandu (Cajanus cajan) cv. Fava Larga, milheto (Pennisetum americanum), mucuna-preta (Mucuna aterrima), soja (Glycine max) cv. FT Estrela, sorgo (Sorghum bicolor) híbrido A-6304, tremoço azul (Lupinus angustifolium) cv. IAPAR 24, e pousio.

Os vasos utilizados constaram da sobreposição de três anéis de PVC, de $10 \mathrm{~cm}$ de diâmetro interno: o superior e o inferior, com $15 \mathrm{~cm}$ de altura cada um, receberam solo com densidade de $1,12 \mathrm{Mg} \mathrm{m}^{-3}$, e o intermediário, com $3,5 \mathrm{~cm}$ de altura, recebeu os tratamentos de compactação.

Para se obter os níveis de compactação, foram adicionadas quantidades determinadas de solo com $8 \%$ de umidade ( $80 \%$ da retenção de água) ao anel de volume conhecido, e em seguida o anel sofreu golpes sucessivos de uma massa de ferro, até atingir os valores de densidade do solo desejados.

Após a montagem, os vasos foram pesados, com a finalidade de manter a umidade inicial durante a condução do experimento, e o controle foi feito com pesagens diárias e regas pela superfície. Foram semeadas as sete espécies citadas, e conservou-se um tratamento sem planta.

Cultivou-se uma planta por vaso de guandu, mucunapreta, soja e tremoço azul, duas plantas de sorgo, e três plantas de aveia-preta, e milheto. Aos 18 dias após a emergência, constatou-se deficiência de $\mathrm{N}$ em algumas plantas, e, em vista disso, realizou-se uma adubação com $50 \mathrm{mg} \mathrm{dm}^{-3}$ de $\mathrm{N}$ em todos os vasos.

Após a emergência (37 a 39 dias), as plantas foram cortadas na altura do colo, picadas em partículas com aproximadamente $3 \mathrm{~cm}$, e deixadas na superfície dos vasos por 40 dias. Nesse intervalo, os vasos foram mantidos com umidade a $80 \%$ da capacidade de retenção de água, para facilitar a decomposição das raízes.

Após esse período, foi realizada a semeadura da soja com sementes pré-germinadas durante três dias em rolo de papel, sem nenhuma adubação adicional. Fez-se o desbaste aos dois dias após a emergência, deixando-se uma planta por vaso.

A colheita das plântulas da soja se deu aos 28 dias após a semeadura, e foram feitas avaliações de produção de matéria seca, concentração e acúmulo dos macronutrientes (Malavolta et al., 1989) da parte aérea das plântulas.

Os resultados foram submetidos à análise de variância, e as médias comparadas pelo teste LSD, a 5\% de probabilidade. 


\section{Resultados e Discussão}

A compactação do solo não causou alteração na produção de matéria seca da parte aérea da soja (Tabela 1). Resultados semelhantes foram encontrados por Rosolem et al. (1994) e Fernandez et al. (1995), ou seja: densidades do solo até $1,72 \mathrm{Mg} \mathrm{m}^{-3}$ não afetaram o desenvolvimento da parte aérea da soja. Tanto naqueles trabalhos, como neste, as plantas foram colhidas entre 28 e 40 dias após a emergência, porém este tempo pode não ter sido suficiente para que os dados de produção de matéria seca apresentassem diferenças entre os níveis de densidade do solo. O cultivo anterior de qualquer uma das espécies favoreceu mais a produção de matéria seca da parte aérea da soja, em comparação com o cultivo após o pousio.

Houve efeito da interação das espécies com as densidades do solo na concentração de N, P, Mg e S, enquanto na de $\mathrm{K}$ houve efeito das espécies e das densidades, mas não da interação entre eles, e apenas a concentração de $\mathrm{Ca}$ foi afetada pelas espécies (Tabela 2).

Quando a soja foi cultivada sobre resíduos de aveia-preta e de milheto, não houve influência dos

Tabela 1. Produção de matéria seca da parte aérea da soja em razão do cultivo anterior e da densidade do solo ${ }^{(1)}$.

\begin{tabular}{lc}
\hline Tratamento & Matéria seca $\left(\mathrm{mg} \mathrm{vaso}^{-1}\right)$ \\
\hline Cultivo anterior & \\
Aveia-preta & $95 \mathrm{abc}$ \\
Guandu & $102 \mathrm{ab}$ \\
Milheto & $86 \mathrm{~cd}$ \\
Mucuna-preta & $95 \mathrm{abc}$ \\
Soja & $93 \mathrm{bcd}$ \\
Sorgo & $82 \mathrm{~d}$ \\
Tremoço azul & $105 \mathrm{a}$ \\
Pousio & $66 \mathrm{e}$ \\
\hline Densidade do solo $\left(\mathrm{Mg} \mathrm{m}^{-3}\right)$ & \\
1,12 & $95 \mathrm{a}$ \\
1,36 & $88 \mathrm{a}$ \\
1,60 & $89 \mathrm{a}$ \\
\hline Fator de variação (teste F) & $*$ \\
Espécies (Es) & $\mathrm{ns}$ \\
Densidades (D) & $\mathrm{ns}$ \\
Es x D & 15,27 \\
\hline CV(\%)
\end{tabular}

(1)Médias seguidas de letras iguais não diferem entre si, pelo teste LSD, a 5\%

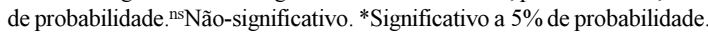

níveis de densidade do solo na concentração desses nutrientes, mas quando cultivada sobre resíduos de guandu, as maiores densidades promoveram aumentos na concentração de $\mathrm{N}$ e de $\mathrm{S}$ (Tabela 3 ). Na menor densidade $\left(1,12 \mathrm{Mg} \mathrm{m}^{-3}\right)$, de modo geral, as leguminosas promoveram maior concentração de $\mathrm{N}$ e de $\mathrm{P}$, e só não diferiram do sorgo. $\mathrm{O}$ tremoço azul promoveu a maior concentração de $\mathrm{Mg}$, e, juntamente com o sorgo, a de $\mathrm{S}$. $\mathrm{Na}$ densidade de $1,36 \mathrm{Mg} \mathrm{m}^{-3}$, o guandu proporcionou maior concentração de $\mathrm{N} \mathrm{e}$ de $\mathrm{P}$, enquanto no $\mathrm{Mg}$ obteve-se a menor concentração quando a soja foi cultivada sobre resíduos de mucuna-preta, embora não tendo diferença em relação ao cultivo sobre resíduos de milheto. A concentração de $\mathrm{S}$ foi maior quando a soja foi cultivada após o tremoço azul e o guandu. Na densidade de $1,60 \mathrm{Mg} \mathrm{m}^{-3}$, a soja teve menor concentração de $\mathrm{N}$ quando foi cultivada após milheto, tremoço azul e pousio. A concentração de $\mathrm{P}$ foi maior na soja após guandu, mucuna-preta e tremoço azul; a de $\mathrm{Mg}$ foi maior após guandu e sorgo, e a de $\mathrm{S}$ foi maior após sorgo e tremoço azul.

De maneira geral, como foi comentado, as leguminosas promoveram maior concentração de $\mathrm{N}$ na parte aérea da soja. Vários autores têm destacado os efeitos de leguminosas no aumento da disponibilidade de $\mathrm{N}$ nas plantas cultivadas posteriormente (Mascarenhas et al., 1978; Gallo et al., 1981; Bayer \& Mielniczuk, 1997); isto acontece por serem espécies fixadoras de $\mathrm{N}$ atmosférico. No presente expe-

Tabela 2. Análise de variância dos valores de concentração e acúmulo de $\mathrm{N}, \mathrm{P}, \mathrm{K}, \mathrm{Ca}, \mathrm{Mg}$ e S na parte aérea da soja cultivada após aveia-preta, guandu, milheto, mucunapreta, soja, sorgo, tremoço azul e pousio.

\begin{tabular}{lcccccc}
\hline Fator de variação & $\mathrm{N}$ & $\mathrm{P}$ & $\mathrm{K}$ & $\mathrm{Ca}$ & $\mathrm{Mg}$ & $\mathrm{S}$ \\
\hline Espécies (Es) & $*$ & $*$ & $*$ & $*$ & $*$ & $*$ \\
Densidades (D) & $*$ & $*$ & $*$ & $\mathrm{~ns}$ & $\mathrm{~ns}$ & $\mathrm{~ns}$ \\
Es x D & $*$ & $*$ & $\mathrm{~ns}$ & $\mathrm{~ns}$ & $*$ & $*$ \\
\hline CV (\%) & 7,61 & 10,25 & 10,70 & 8,62 & 7,31 & 23,04 \\
\hline & $*$ & $*$ & $*$ & $*$ & $*$ & $*$ \\
Espécies (Es) & $*$ & $*$ & $*$ & $*$ & $*$ & $\mathrm{~ns}$ \\
Densidades (D) & $\mathrm{ns}$ & $*$ & $\mathrm{nc}$ & \\
Es x D & $\mathrm{ns}$ & $*$ & $\mathrm{~ns}$ & $\mathrm{~ns}$ & $\mathrm{~ns}$ & $\mathrm{~ns}$ \\
\hline CV (\%) & 13,50 & 18,44 & 16,79 & 18,92 & 15,04 & 34,28 \\
\hline
\end{tabular}

nsNão-significativo. *Significativo, pelo teste LSD, a 5\% de probabilidade. 
rimento, essa maior disponibilidade deveu-se, provavelmente, à rápida decomposição do material vegetal das leguminosas, como decorrência da baixa relação $\mathrm{C} / \mathrm{N}$, determinando, assim, a rápida mineralização do N (Hass, 1999), apesar de nem sempre as diferenças terem sido significativas em relação aos outros resíduos.

A rápida decomposição dos resíduos das leguminosas, exceto a do tremoço azul, também proporcionou maior concentração de $\mathrm{P}$ na soja, e no cultivo após o guandu apresentou maior valor, principalmente na densidade de $1,36 \mathrm{Mg} \mathrm{m}^{-3}$ (Tabela 3). Como a presença de resíduos nem sempre promoveu aumento na concentração de $\mathrm{P}$, em comparação com o pousio, concorda-se que o tempo de decomposição é importante para aumentar a disponibilidade do P para a planta subseqüente (Hass, 1999).

Embora Fernandez et al. (1995) tenham verificado que houve redução do teor de $\mathrm{Mg}$ com o aumento da compactação do solo, no presente experimento não ocorreu o mesmo (Tabela 3), e tal resultado pode ser atribuído à presença de resíduos vegetais na superfície do solo.

Na maior densidade do solo $\left(1,60 \mathrm{Mg} \mathrm{m}^{-3}\right)$, a concentração de $\mathrm{S}$ na soja foi menor após o pousio, embo- ra sem diferença em relação ao cultivo após a mucunapreta. De acordo com Vitti (1989), o cultivo do solo aumenta a mineralização do S orgânico, e, como a maior parte do $\mathrm{S}$ é encontrada no solo na forma orgânica, e sua mineralização é necessária para torná-lo disponível às plantas, é possível que o cultivo das espécies e sua decomposição tenha promovido a mineralização do S, tornando-o disponível para a soja.

Quanto aos nutrientes que não foram influenciados pela interação entre as espécies com as densidades do solo, houve menor concentração de K na parte aérea da soja somente na densidade de $1,36 \mathrm{Mg} \mathrm{m}^{-3}$, em relação à menor densidade do solo. Porém, outros fatores, além da densidade do solo, podem estar interferindo nos resultados, pois não houve diferença significativa entre a maior e a menor densidade do solo (Tabela 4).

A soja cultivada sobre resíduos de aveia-preta apresentou menor concentração de $\mathrm{K}$ na parte aérea em relação às outras espécies e ao pousio (Tabela 5). Não se encontrou explicação de tal resultado em relação ao pousio, pois o K é um dos primeiros elementos liberados na decomposição de resíduos vegetais, principalmente pelo processo de lavagem ou lixiviação (Malavolta, 1980).

Tabela 3. Desdobramento da interação entre as espécies e as densidades do solo na concentração de macronutrientes na parte aérea da soja em razão da densidade do solo e do cultivo anterior ${ }^{(1)}$.

\begin{tabular}{|c|c|c|c|c|c|c|c|c|}
\hline $\begin{array}{l}\text { Densidade do } \\
\text { solo }\left(\mathrm{Mg} \mathrm{m}^{-3}\right)\end{array}$ & Aveia-preta & Guandu & Milheto & Mucuna-preta & Soja & Sorgo & Tremoço azul & Pousio \\
\hline & \multicolumn{8}{|c|}{$\mathrm{N}\left(\mathrm{g} \mathrm{kg}^{-1}\right)$} \\
\hline 1,12 & $36,3 \mathrm{aBCD}$ & $43,2 \mathrm{bA}$ & $35,6 \mathrm{aD}$ & $42,7 \mathrm{aA}$ & $41,0 \mathrm{bAB}$ & $40,2 \mathrm{bABC}$ & $42,9 \mathrm{aA}$ & $36,0 \mathrm{aCD}$ \\
\hline 1,36 & $40,2 \mathrm{aBC}$ & $51,4 \mathrm{aA}$ & $37,2 \mathrm{aB}$ & $44,2 \mathrm{aB}$ & $41,0 \mathrm{bBC}$ & $39,5 \mathrm{bC}$ & $44,5 \mathrm{aB}$ & $34,1 \mathrm{aD}$ \\
\hline \multirow[t]{2}{*}{1,60} & $40,8 \mathrm{aAB}$ & $48,3 \mathrm{aA}$ & $37,8 \mathrm{aD}$ & $43,0 \mathrm{aBC}$ & $46,8 \mathrm{aAB}$ & $46,3 \mathrm{aAB}$ & $41,5 \mathrm{aCD}$ & $32,8 \mathrm{aE}$ \\
\hline & \multicolumn{8}{|c|}{$\mathrm{P}\left(\mathrm{g} \mathrm{kg}^{-1}\right)$} \\
\hline 1,12 & $1,37 \mathrm{aC}$ & $2,18 \mathrm{aA}$ & $1,46 \mathrm{aBC}$ & $1,97 \mathrm{aA}$ & $2,16 \mathrm{aA}$ & $1,65 \mathrm{aB}$ & $1,46 \mathrm{bBC}$ & $1,46 \mathrm{aBC}$ \\
\hline 1,36 & $1,45 \mathrm{aBC}$ & $1,98 \mathrm{aA}$ & $1,32 \mathrm{aC}$ & $1,65 \mathrm{bB}$ & $1,60 \mathrm{bB}$ & $1,32 \mathrm{bC}$ & $1,32 \mathrm{bC}$ & $1,30 \mathrm{aC}$ \\
\hline \multirow[t]{2}{*}{1,60} & $1,29 \mathrm{aD}$ & $2,10 \mathrm{aA}$ & $1,27 \mathrm{aD}$ & $2,04 \mathrm{aAB}$ & $1,68 \mathrm{bC}$ & $1,85 \mathrm{aBC}$ & $2,04 \mathrm{aAB}$ & $1,35 \mathrm{aD}$ \\
\hline & \multicolumn{8}{|c|}{$\mathrm{Mg}\left(\mathrm{g} \mathrm{kg}^{-1}\right)$} \\
\hline 1,12 & $5,78 \mathrm{aB}$ & $6,25 \mathrm{aB}$ & $5,68 \mathrm{aB}$ & $5,85 \mathrm{aB}$ & $5,82 \mathrm{aB}$ & $6,08 \mathrm{bB}$ & $6,92 \mathrm{aA}$ & $6,10 \mathrm{aB}$ \\
\hline 1,36 & $6,12 \mathrm{aAB}$ & $6,32 \mathrm{aA}$ & $5,58 \mathrm{aBC}$ & $5,12 b C$ & $5,88 \mathrm{aAB}$ & $6,22 \mathrm{bA}$ & $6,25 \mathrm{bA}$ & $6,32 \mathrm{aA}$ \\
\hline \multirow[t]{2}{*}{1,60} & $5,85 \mathrm{aC}$ & $6,58 \mathrm{aAB}$ & $5,50 \mathrm{aC}$ & $5,98 \mathrm{aBC}$ & $6,02 \mathrm{aBC}$ & $7,20 \mathrm{aA}$ & $6,52 \mathrm{abB}$ & $5,98 \mathrm{aBC}$ \\
\hline & \multicolumn{8}{|c|}{$\mathrm{S}\left(\mathrm{g} \mathrm{kg}^{-1}\right)$} \\
\hline 1,12 & $1,67 \mathrm{aBC}$ & $1,59 \mathrm{bBC}$ & $1,32 \mathrm{aC}$ & $1,55 \mathrm{aBC}$ & $1,49 \mathrm{aBC}$ & $2,49 \mathrm{aA}$ & $2,03 \mathrm{aAB}$ & $1,39 \mathrm{aC}$ \\
\hline 1,36 & $1,47 \mathrm{aCD}$ & $2,16 \mathrm{aAB}$ & $1,49 \mathrm{aCD}$ & $1,28 \mathrm{aDE}$ & $1,85 \mathrm{aBC}$ & $1,89 \mathrm{bBC}$ & $2,54 \mathrm{aA}$ & $0,89 \mathrm{abE}$ \\
\hline 1,60 & $1,56 \mathrm{aBCD}$ & $1,84 \mathrm{abBC}$ & $1,47 \mathrm{aCD}$ & $1,14 \mathrm{aDE}$ & $1,87 \mathrm{aBC}$ & $2,52 \mathrm{aA}$ & $2,06 \mathrm{aAB}$ & $0,69 \mathrm{bE}$ \\
\hline
\end{tabular}

\footnotetext{
${ }^{(1)}$ Médias seguidas das mesmas letras, maiúsculas nas linhas e minúsculas nas colunas, não diferem entre si, pelo teste LSD, a 5\% de probabilidade.
} 
A concentração de Ca na parte aérea da soja cultivada sobre resíduos de mucuna-preta e sorgo foi menor em relação ao cultivo após o guandu; porém, este não diferiu do cultivo sobre os outros resíduos e após o pousio. Santos \& Tomm (1996) observaram que, em geral, os sistemas de rotação de culturas com trigo não proporcionaram diferenças significativas entre as médias de Ca trocável do solo. Gediga (1991) sugeriu que isto, provavelmente, ocorre porque a maior parte do $\mathrm{Ca}$ é absorvida da zona nãocompactada. No presente caso, embora não tenha havido influência das densidades do solo, o mesmo efeito pode ter ocorrido.

Tabela 4. Concentração de $\mathrm{K}$ na parte aérea da soja em razão da densidade do solo ${ }^{(1)}$.

\begin{tabular}{cc}
\hline Densidade do solo $\left(\mathrm{Mg} \mathrm{m}^{-3}\right)$ & $\mathrm{K}\left(\mathrm{g} \mathrm{kg}^{-1}\right)$ \\
\hline 1,12 & $16,14 \mathrm{a}$ \\
1,36 & $15,03 \mathrm{~b}$ \\
1,60 & $15,74 \mathrm{ab}$ \\
\hline
\end{tabular}

${ }^{(1)}$ Médias seguidas de letras iguais, nas colunas, não diferem entre si, pelo teste $\mathrm{LSD}$, a $5 \%$ de probabilidade.

Tabela 5. Concentração $\left(\mathrm{g} \mathrm{kg}^{-1}\right)$ de $\mathrm{K}$ e de Ca na parte aérea da soja em razão do cultivo anterior ${ }^{(1)}$.

\begin{tabular}{lll}
\hline Cultivo anterior & $\mathrm{K}$ & $\mathrm{Ca}$ \\
\hline Aveia-preta & $13,08 \mathrm{~b}$ & $22,68 \mathrm{ab}$ \\
Guandu & $15,60 \mathrm{a}$ & $23,00 \mathrm{a}$ \\
Milheto & $15,88 \mathrm{a}$ & $22,00 \mathrm{abc}$ \\
Mucuna-preta & $16,52 \mathrm{a}$ & $21,04 \mathrm{c}$ \\
Soja & $16,31 \mathrm{a}$ & $21,66 \mathrm{abc}$ \\
Sorgo & $15,82 \mathrm{a}$ & $21,37 \mathrm{bc}$ \\
Tremoço-azul & $16,30 \mathrm{a}$ & $22,70 \mathrm{ab}$ \\
Pousio & $15,60 \mathrm{a}$ & $22,18 \mathrm{abc}$ \\
\hline
\end{tabular}

(1)Médias seguidas de letras iguais, nas colunas, não diferem entre si, pelo teste LSD, a $5 \%$ de probabilidade.
Com a finalidade de eliminar o efeito diluição, analisou-se o acúmulo dos nutrientes na matéria seca da parte aérea da planta, no qual se verificou efeito da interação entre cultivo anterior e densidade do solo sobre o acúmulo de $\mathrm{P}$ (Tabela 6). Os outros nutrientes foram influenciados pelas espécies anteriores e, exceto $\mathrm{N}$ e S, também pela densidade do solo (Tabela 2).

$\mathrm{Na}$ soja cultivada em resíduos de aveia-preta e após o pousio, o aumento da densidade do solo não provocou variação no acúmulo de $\mathrm{P}$, enquanto sobre os resíduos de guandu, milheto e soja, houve menor acúmulo nas maiores densidades (Tabela 6). Sobre os resíduos de tremoço azul, ocorreu maior acúmulo do $\mathrm{P}$ na soja, quando na densidade de $1,60 \mathrm{Mg} \mathrm{m}^{-3}$.

O cultivo da soja após o pousio apresentou menor acúmulo de $\mathrm{N}, \mathrm{K}, \mathrm{Ca}, \mathrm{Mg}$ e $\mathrm{S}$, mostrando, assim, que o cultivo anterior promoveu maior eficiência no acúmulo dos nutrientes na parte aérea da planta (Tabela 7). Muzilli (1985) também observou que o cultivo de várias espécies pode determinar mudanças nas propriedades químicas do solo, e os efeitos se refletem diretamente na fertilidade e na eficiência de aproveitamento de nutrientes pelas plantas.

As leguminosas proporcionaram maior acúmulo de nutrientes na parte aérea da soja, o que permite concluir que não houve efeito concentração/diluição dos nutrientes na planta, ou seja, houve utilização dos nutrientes absorvidos na produção de matéria seca da planta.

Quanto ao efeito da densidade do solo (Tabela 8), houve redução no acúmulo de $\mathrm{K}, \mathrm{Ca}$ e $\mathrm{Mg}$ quando a densidade aumentou de 1,12 para $1,36 \mathrm{Mg} \mathrm{m}^{-3}$. De acordo com Marschner (1986), dependendo do tipo de solo e da umidade, em densidades do solo de até $1,40 \mathrm{Mg} \mathrm{m}^{-3}$ não há inibição na absorção de nutrientes. Porém, no presente caso, a densidade de

Tabela 6. Desdobramento da interação entre as espécies e as densidades do solo no acúmulo de P na matéria seca da parte aérea da soja em razão do cultivo anterior e das densidades do solo ${ }^{(1)}$.

\begin{tabular}{|c|c|c|c|c|c|c|c|c|}
\hline \multirow[t]{2}{*}{ Densidade do solo } & \multicolumn{8}{|c|}{ Cultivo anterior } \\
\hline & Aveia-preta & Guandu & Milheto & Mucuna-preta & Soja & Sorgo & Tremoço azul & Pousio \\
\hline$\left(\mathrm{Mg} \mathrm{m}^{-3}\right)$ & & 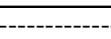 & 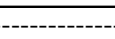 & ----P (mg & aso $\left.^{-1}\right)--$ & -------- & 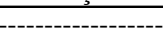 & 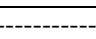 \\
\hline 1,12 & $1,45 \mathrm{aCD}$ & $2,68 \mathrm{aA}$ & $1,38 \mathrm{aD}$ & $1,80 \mathrm{abBC}$ & $2,15 \mathrm{aB}$ & $1,35 \mathrm{abD}$ & $1,45 b C D$ & $0,95 \mathrm{aE}$ \\
\hline 1,36 & $1,32 \mathrm{aBC}$ & $1,88 \mathrm{bA}$ & $1,12 \mathrm{bC}$ & $1,55 \mathrm{bAB}$ & $1,52 \mathrm{bAB}$ & $1,05 \mathrm{bCD}$ & $1,28 \mathrm{bBC}$ & $0,85 \mathrm{aD}$ \\
\hline 1,60 & $1,15 \mathrm{aDEF}$ & $1,90 \mathrm{bBC}$ & $1,05 \mathrm{bEF}$ & $1,98 \mathrm{aB}$ & $1,40 \mathrm{bDE}$ & $1,52 \mathrm{aCD}$ & $2,45 \mathrm{aA}$ & $0,90 \mathrm{aF}$ \\
\hline
\end{tabular}

${ }^{(1)}$ Médias seguidas das mesmas letras, maiúsculas nas linhas e minúsculas nas colunas, não diferem entre si, pelo teste LSD, a 5\% de probabilidade. 
Tabela 7. Acúmulo (mg vaso ${ }^{-1}$ ) de N, K, Ca, Mg e S na matéria seca da parte aérea da soja em razão do cultivo anterior $^{(1)}$.

\begin{tabular}{llllll}
\hline Cultivo anterior & \multicolumn{1}{c}{$\mathrm{N}$} & \multicolumn{1}{c}{$\mathrm{K}$} & \multicolumn{1}{c}{$\mathrm{Ca}$} & $\mathrm{Mg}$ & $\mathrm{S}$ \\
\hline Aveia-preta & $37 \mathrm{bc}$ & $12,50 \mathrm{~cd}$ & $21,46 \mathrm{ab}$ & $5,62 \mathrm{~b}$ & $1,50 \mathrm{~cd}$ \\
Guandu & $48 \mathrm{a}$ & $15,92 \mathrm{ab}$ & $23,82 \mathrm{a}$ & $6,52 \mathrm{a}$ & $1,89 \mathrm{~b}$ \\
Milheto & $32 \mathrm{~d}$ & $13,76 \mathrm{bc}$ & $18,98 \mathrm{bc}$ & $4,83 \mathrm{c}$ & $1,22 \mathrm{~d}$ \\
Mucuna-preta & $41 \mathrm{~b}$ & $15,58 \mathrm{ab}$ & $19,72 \mathrm{bc}$ & $5,32 \mathrm{bc}$ & $1,24 \mathrm{~d}$ \\
Soja & $40 \mathrm{~b}$ & $15,28 \mathrm{ab}$ & $20,18 \mathrm{~b}$ & $5,50 \mathrm{bc}$ & $1,62 \mathrm{bc}$ \\
Sorgo & $34 \mathrm{~cd}$ & $12,90 \mathrm{c}$ & $17,42 \mathrm{c}$ & $5,31 \mathrm{bc}$ & $1,86 \mathrm{~b}$ \\
Tremoço azul & $41 \mathrm{~b}$ & $17,19 \mathrm{a}$ & $23,67 \mathrm{a}$ & $6,88 \mathrm{a}$ & $2,30 \mathrm{a}$ \\
Pousio & $23 \mathrm{e}$ & $10,41 \mathrm{~d}$ & $14,68 \mathrm{~d}$ & $4,04 \mathrm{~d}$ & $0,65 \mathrm{e}$ \\
\hline
\end{tabular}

${ }^{(1)}$ Médias seguidas de letras iguais, nas colunas, não diferem entre si, pelo teste LSD, a $5 \%$ de probabilidade.

Tabela 8. Acúmulo de K, Ca e Mg na matéria seca da parte aérea da soja em razão das densidades do solo(1).

\begin{tabular}{cccc}
\hline $\begin{array}{c}\text { Densidade do solo } \\
\left(\mathrm{Mg} \mathrm{m}^{-3}\right)\end{array}$ & \multicolumn{1}{c}{$\mathrm{K}$} & $\begin{array}{c}\mathrm{Ca} \\
\mathrm{Mg}\end{array}$ & $\left.\mathrm{Mg}{ }^{-1}\right)$ \\
\hline 1,12 & $15,34 \mathrm{a}$ & $21,21 \mathrm{a}$ & $5,73 \mathrm{a}$ \\
1,36 & $13,19 \mathrm{~b}$ & $19,41 \mathrm{~b}$ & $5,22 \mathrm{~b}$ \\
1,60 & $14,05 \mathrm{ab}$ & $19,35 \mathrm{~b}$ & $5,59 \mathrm{ab}$ \\
\hline
\end{tabular}

${ }^{(1)}$ Médias seguidas de letras iguais, nas colunas, não diferem entre si, pelo teste LSD, a $5 \%$ de probabilidade.

1,36 $\mathrm{Mg} \mathrm{m}^{-3}$ foi suficiente para diminuir o acúmulo de K, Ca e Mg pela soja, independentemente do cultivo anterior.

\section{Conclusões}

1. A compactação do solo em subsuperfície diminui a nutrição da soja.

2. A nutrição da soja é beneficiada quando cultivada em sucessão a plantas de cobertura no solo.

3. O pousio antecedendo a cultura da soja não reduz os efeitos da compactação do solo.

\section{Referências}

BAYER, C.; MIELNICZUK, J. Nitrogênio total de um solo submetido a diferentes métodos de preparo e sistemas de cultura. Revista Brasileira de Ciência do Solo, Campinas, v. 21, p. 235-239, 1997.

BENGOUGH, A. G.; YOUNG, I. M. Root elongation of seedling peas through layered soil of different penetration resistances. Plant and Soil, v. 149, p. 129-139, 1993.

FERNANDEZ, E. M.; CRUSCIOL, C. A. C.; THIMOTHEO, C. M. S.; ROSOLEM, C. A. Matéria seca e nutrição da soja em razão da compactação do solo e adubação fosfatada. Científica, Jaboticabal, v. 23, n. 1, p. 117-132, 1995.

FIORIN, J. E. Plantas recuperadoras da fertilidade do solo. In: CURSO sobre aspectos básicos de fertilidade e microbiologia do solo em plantio direto: resumos de palestras. Passo Fundo: Aldeia Norte, 1999. p. 39-55.

GALLO, P. B.; LAVORENTI, A.; SAWAZAKI, E.; HIROCE, R.; MASCARENHAS, H. A. A. Efeito de cultivos de soja na produção e no teor de nitrogênio das folhas e dos grãos de milho. Revista Brasileira de Ciência do Solo, Campinas, v. 5, p. 64-67, 1981.

GEDIGA, K. Influence of subsoil compaction on the uptake of ${ }^{45} \mathrm{Ca}$ from the soil profile and on maize yield. Soil and Tillage Research, Amsterdam, v. 19, p. 351-355, 1991.

HASS, F. D. Aspectos básicos de fertilidade sob plantio direto. In: CURSO sobre aspectos básicos de fertilidade e microbiologia do solo em plantio direto: resumos de palestras. Passo Fundo: Aldeia Norte, 1999. p. 19-31.

JESSOP, R. S.; STEWART, L. W. Effects of crops residue, soil type and temperature on emergence and early growth of wheat. Plant and Soil, Dordrecht, v. 74, p. 101-109, 1983.

LYNCH, J. M. Interactions between biological processes, cultivation and soil structure. Plant and Soil, Dordrecht, v. 76, p. 307-318, 1984.

MALAVOLTA, E. Elementos de nutrição mineral de plantas. São Paulo: Agronômica Ceres, 1980. 251 p.

MALAVOLTA, E.; VITTI, G. C.; OLIVEIRA, S. A. Avaliação do estado nutricional de plantas: princípios e aplicações. Piracicaba: Potafos, 1989. 201 p.

MARSCHNER, H. Mineral nutrition of higher plants. New York: Academic, 1986. 403 p.

MASCARENHAS, H. A. A.; HIROCE, R.; BRAGA, N. R.; MIRANDOA, M. A. C.; POMMER, C. V.; SAWAZAKI, E. Efeito do nitrogênio residual de soja na produção de milho. Campinas: Instituto Agronômico, 1978. 16 p. (Boletim Técnico, 58).

MUZILLI, O. Fertilidade do solo em plantio direto. In: FANCELLI, A. L.; TORRADO, P. V.; MACHADO, J. (Ed.). Atualização em plantio direto. Campinas: Fundação Cargill, 1985. p. 147-160. 
PINTO, L. F. G.; CRESTANA, S. Viabilidade do uso da adubação verde nos agroecossistemas da região de São Paulo, SP. Revista Brasileira de Ciência do Solo, Viçosa, v. 22, p. 329-336, 1998.

ROSOLEM, C. A.; ALMEIDA, A. C. S.; SACRAMENTO, L. V. S. Sistema radicular e nutrição da soja em função da compactação do solo. Bragantia, Campinas, v. 53, n. 2, p. 259-266, 1994.

SANTOS, H. P.; TOMM, G. O. Estudo da fertilidade do solo sob quatro sistemas de rotação de culturas envolven- do trigo em plantio direto. Revista Brasileira de Ciência do Solo, Campinas, v. 20, p. 407-414, 1996.

SILBERBUSH, M.; HALLMARK, W. B.; BARBER, S. A. Simulation of effects of soil bulk density and $\mathrm{P}$ addition on $\mathrm{K}$ uptake of soybean. Communications in Soil Science and Plant Analysis, New York, v. 14, p. 287-296, 1983.

VITTI, G. C. Enxofre no solo. In: BÜLL, L. T.; ROSOLEM, C. A. (Ed.). Interpretação de análise química de solo e planta para fins de adubação. Botucatu: Fepaf, 1989. 360 p. 